\title{
Universally valid reformulation of the Heisenberg uncertainty principle on noise and disturbance in measurement
}

\author{
Masanao Ozawa \\ Graduate School of Information Sciences, Tôhoku University, Aoba-ku, Sendai, 980-8579, Japan
}

The Heisenberg uncertainty principle 但 states that the product of the noise in a position measurement and the momentum disturbance caused by that measurement should be no less than the limit set by Planck's constant, $\hbar / 2$, as demonstrated by Heisenberg's thought experiment using a $\gamma$-ray microscope [2]. Here I show that this common assumption is false: a universally valid trade-off relation between the noise and the disturbance has an additional correlation term, which is redundant when the intervention brought by the measurement is independent of the measured object, but which allows the noise-disturbance product much below Planck's constant when the intervention is dependent. A model of measuring interaction with dependent intervention shows that Heisenberg's lower bound for the noise-disturbance product is violated even by a nearly nondisturbing, precise position measuring instrument. An experimental implementation is also proposed to realize the above model in the context of optical quadrature measurement with currently available linear optical devices.

PACS numbers: 03.65.Ta, 04.80.Nn, 03.67.-a

\section{THREE FORMULATIONS OF UNCERTAINTY PRINCIPLE}

The uncertainty principle has been known as one of the most fundamental principles in quantum mechanics. However, there is still ambiguity in formulation; we have at least three different formulations of the "uncertainty principle".

The Robertson uncertainty relation is generally formulated as the relation

$$
\sigma(A, \psi) \sigma(B, \psi) \geq \frac{|\langle\psi|[A, B]| \psi\rangle|}{2}
$$

for any observables $A, B$ and any state $\psi$, where the standard deviation $\sigma(X, \psi)$ of an observable $X$ in state $\psi$ is defined by $\sigma(X, \psi)^{2}=\left\langle\psi\left|X^{2}\right| \psi\right\rangle-\langle\psi|X| \psi\rangle^{2}$. This relation was proven mathematically from fundamental postulates of quantum mechanics [3, 4]. Nevertheless, this relation describes the limitation on preparing microscopic objects but has no direct relevance to the limitation of accuracy of measuring devices [5].

It is a common understanding that the uncertainty principle implies or is implied by a limitation on measuring a system without disturbing it as a position measurement typically disturbs the momentum. However, the limitation has eluded a correct quantitative expression on the trade-off between noise and disturbance. By the $\gamma$-ray thought experiment, Heisenberg [1, 2] argued that the product of the noise in a position measurement and the momentum disturbance caused by that measurement should be no less than $\hbar / 2$. This relation is generally formulated as follows: For any apparatus $\mathbf{A}$ to measure an observable $A$, the relation

$$
\epsilon(A, \psi, \mathbf{A}) \eta(B, \psi, \mathbf{A}) \geq \frac{|\langle\psi|[A, B]| \psi\rangle|}{2}
$$

holds for any input state $\psi$ and any observable $B$, where $\epsilon(A, \psi, \mathbf{A})$ stands for the noise of the $A$ measurement in state $\psi$ using apparatus $\mathbf{A}$ and $\eta(B, \psi, \mathbf{A})$ stands for the disturbance of $B$ in state $\psi$ caused by apparatus $\mathbf{A}$. We refer to the above relation as the Heisenberg noisedisturbance uncertainty relation. We shall investigate the validity of this relation to solve such questions as: When does this relation hold and when does not? What relation can be considered a universally valid generalization of this relation? How can we experimentally observe the violation of this relation?

Closely related to the above relation, the Heisenberg uncertainty relation for joint measurements is generally formulated as follows: For any apparatus $\mathbf{A}$ with two outputs for the joint measurement of $A$ and $B$, the relation

$$
\epsilon(A, \psi, \mathbf{A}) \epsilon(B, \psi, \mathbf{A}) \geq \frac{|\langle\psi|[A, B]| \psi\rangle|}{2}
$$

holds for any input state $\psi$, where $\epsilon(X, \psi, \mathbf{A})$ stands for the noise of the $X$ measurement in state $\psi$ using apparatus $\mathbf{A}$ for $X=A, B$. This relation was proven mathematically [6] under the joint unbiasedness condition requiring that the (experimental) mean values of the outcome $\mathbf{x}$ of the $A$ measurement and the outcome $\mathbf{y}$ of the $B$ measurement should coincide with the (theoretical) mean values of observables $A$ and $B$, respectively, on any input state $\psi$. It is a common opinion that currently available measuring devices satisfy this relation [10]12].

There is a logical relationship between the noisedisturbance relation, (2), and the joint measurement relation, (3). Suppose that the $A$ measurement using the apparatus $\mathbf{A}$ is followed immediately by a measurement of the observable $B$ using a noiseless measuring apparatus B. Then, combining the two apparatuses, we have a new apparatus $\mathbf{C}$ to jointly measure $A$ and $B$ on the input state of apparatus A. Since $\mathbf{B}$ carries out a noiseless measurement on its own input state, the noise of $B$ measurement in the outcome of apparatus $\mathbf{C}$ is equal to the $B$ disturbance caused by apparatus A. Thus, we have the relations 


$$
\begin{aligned}
\epsilon(A, \psi, \mathbf{A}) & =\epsilon(A, \psi, \mathbf{C}), \\
\eta(B, \psi, \mathbf{A}) & =\epsilon(B, \psi, \mathbf{C}) .
\end{aligned}
$$

By applying the uncertainty relation for joint measurements to the apparatus $\mathbf{C}$, we can conclude that the noise-disturbance relation, (2), holds if apparatus $\mathbf{C}$ satisfies the joint unbiasedness condition for observables $A$ and $B$. However, there are two deficiencies of the above approach: (i) Even for noiseless measuring apparatus A to measure $A$ one cannot ensure that the combined apparatus $\mathbf{C}$ satisfies the joint unbiasedness condition. (ii) The above argument does not give a universally valid trade-off relation between noise and disturbance. Thus, we can conclude that the validity of the noise-disturbance relation, (2), cannot be reduced to the current formulation of the Heisenberg uncertainty relation for joint measurements, (3).

\section{MEASUREMENT NOISE AND DISTURBANCE}

Now, let us analyze noise and disturbance in a most general description of measurement 13 15 in detail. Let A be a measuring apparatus with (macroscopic) output variable $\mathbf{x}$ to measure an observable $A$ of a quantum system $\mathbf{S}$. Then, apparatus $\mathbf{A}$ measures observable $A$ precisely if and only if the (experimental) probability distribution of $\mathrm{x}$ coincides with the (theoretical) probability distribution of $A$; or rigorously the probability of the event that the output $\mathbf{x}$ of apparatus $\mathbf{A}$ is in an interval $\Delta$ satisfies the Born statistical formula (BSF) for observable $A$, i.e.,

$$
\operatorname{Pr}\{\mathbf{x} \in \Delta\}=\left\langle\psi\left|E^{A}(\Delta)\right| \psi\right\rangle
$$

on every input state $\psi$, where $E^{A}(\Delta)$ stands for the spectral projection of $A$ corresponding to interval $\Delta$. Otherwise, we consider apparatus $\mathbf{A}$ to measure observable $A$ with some noise. In order to evaluate the noise, we need to describe the measuring process. The measuring interaction is supposed to turn on at a time $t$, the time of measurement, and to turn off at time $t+\Delta t$ between the object $\mathbf{S}$ and a quantum subsystem $\mathbf{P}$, called the probe, of the (large) apparatus A. Denote by $U$ the unitary operator representing the time evolution of the composite system $\mathbf{S}+\mathbf{P}$ for the time interval $(t, t+\Delta t)$. We assume that the object and any part of the apparatus do not interact before $t$ nor after $t+\Delta t$. At the time of measurement the object is supposed to be in an arbitrary state $\psi$ and the probe is in a fixed state $\xi$. Thus, the composite system $\mathbf{S}+\mathbf{P}$ is in the state $\psi \otimes \xi$ at time $t$. Just after the measuring interaction has turned off, the probe is subjected to a precise local measurement of an observable $M$ of the probe, called the probe observable, and its output is recorded by the output variable $\mathbf{x}$. In the Heisenberg picture with the original state $\psi \otimes \xi$ at time $t$, we write $A^{\text {in }}=A \otimes I, M^{\text {in }}=I \otimes M$,
$A^{\text {out }}=U^{\dagger}(A \otimes I) U$, and $M^{\text {out }}=U^{\dagger}(I \otimes M) U$. Since the output of this measurement is obtained by the precise measurement of observable $M$ at time $t+\Delta t$, the probability distribution of the output variable $\mathbf{x}$ is given by

$$
\operatorname{Pr}\{\mathbf{x} \in \Delta\}=\left\langle E^{M^{\text {out }}}(\Delta)\right\rangle,
$$

where $\langle\cdots\rangle$ stands for $\langle\psi \otimes \xi|\cdots| \psi \otimes \xi\rangle$ throughout this paper.

The noise $\epsilon(A, \psi, \mathbf{A})$, or $\epsilon(A)$ for short, of the $A$ measurement on input state $\psi$ using apparatus $\mathbf{A}$ is defined as the root-mean-square deviation of the experimental variable $M^{\text {out }}$ from the theoretical variable $A^{\text {in }}$, i.e.,

$$
\epsilon(A, \psi, \mathbf{A})=\left\langle\left(M^{\text {out }}-A^{\text {in }}\right)^{2}\right\rangle^{1 / 2} .
$$

Then, we can prove that $\epsilon(A)=0$ on any input state $\psi$ if and only if apparatus $\mathbf{A}$ measures observable A precisely. This ensures that "precise apparatuses" and "noiseless apparatuses" are equivalent. Let $B$ be an observable of S.

The disturbance $\eta(B, \psi, \mathbf{A})$, or $\eta(B)$ for short, of observable $B$ on input state $\psi$ caused by apparatus $\mathbf{A}$ is defined as the root-mean-square of the change in the observable $B$ during the measuring interaction, i.e.,

$$
\eta(B, \psi, \mathbf{A})=\left\langle\left(B^{\text {out }}-B^{\text {in }}\right)^{2}\right\rangle^{1 / 2} .
$$

Then, we can prove that $\eta(B)=0$ on any input state $\psi$ if and only if apparatus $\mathbf{A}$ does not change the probability distribution of the observable $B$, i.e., $\left\langle E^{B^{\text {in }}}(\Delta)\right\rangle=$ $\left\langle E^{B^{\text {out }}}(\Delta)\right\rangle$ for every interval $\Delta$ on any input state $\psi$. Thus, apparatuses not disturbing the observable $B$ and apparatuses with zero disturbance, $\eta(B) \equiv 0$, are equivalent notions. It should be also noted that the above definitions of noise and disturbance are consistent with the standard formulation for the Heisenberg uncertainty relation for joint measurements, Eq. (3), with Eq. (4). Thus, the above definitions of noise and disturbance can be considered standard.

\section{UNIVERSALLY VALID UNCERTAINTY PRINCIPLE}

Under the above general definitions of noise and disturbance, we can rigorously investigate the validity of the Heisenberg noise-disturbance uncertainty relation, Eq. (2). For this purpose, we introduce the noise operator $N(A)$ and the disturbance operator $D(B)$ by

$$
\begin{aligned}
M^{\text {out }} & =A^{\text {in }}+N(A), \\
B^{\text {out }} & =B^{\text {in }}+D(B) .
\end{aligned}
$$

Since $M$ and $B$ are observables in different systems, we have $\left[M^{\text {out }}, B^{\text {out }}\right]=0$, and hence we have the following commutation relation for the noise operator and the disturbance operator, 


$$
\begin{aligned}
& {[N(A), D(B)]+\left[N(A), B^{\text {in }}\right]+\left[A^{\text {in }}, D(B)\right]} \\
& \quad=-\left[A^{\text {in }}, B^{\text {in }}\right] .
\end{aligned}
$$

Taking the modulus of means of the both sides and applying the triangular inequality, we have

$$
\begin{aligned}
& |\langle[N(A), D(B)]\rangle|+\left|\left\langle\left[N(A), B^{\text {in }}\right]\right\rangle+\left\langle\left[A^{\text {in }}, D(B)\right]\right\rangle\right| \\
& \quad \geq|\langle\psi|[A, B]| \psi\rangle| .
\end{aligned}
$$

Since the variance is not greater than the mean square, we have

$$
\begin{aligned}
\epsilon(A, \psi, \mathbf{A}) & \geq \sigma(N(A), \psi \otimes \sigma), \\
\eta(B, \psi, \mathbf{A}) & \geq \sigma(D(B), \psi \otimes \sigma),
\end{aligned}
$$

and hence by the Heisenberg uncertainty principle, Eq. (11), we have

$$
\epsilon(A) \eta(B) \geq \frac{|\langle[N(A), D(B)]\rangle|}{2} .
$$

Thus, we obtain the universally valid noise-disturbance uncertainty relation for the pair $(A, B)$,

$$
\begin{aligned}
\epsilon(A) & \eta(B)+\frac{\left|\left\langle\left[N(A), B^{\mathrm{in}}\right]\right\rangle+\left\langle\left[A^{\mathrm{in}}, D(B)\right]\right\rangle\right|}{2} \\
& \geq \frac{|\langle\psi|[A, B]| \psi\rangle|}{2} .
\end{aligned}
$$

The above relation shows that the Heisenberg noisedisturbance uncertainty relation, Eq. (2), holds if the correlation term $\left|\left\langle\left[N(A), B^{\text {in }}\right]\right\rangle+\left\langle\left[A^{\text {in }}, D(B)\right]\right\rangle\right|$ vanishes. In order to characterize a class of measurements satisfying Eq. (2), we introduce the following definition. The measuring interaction is said to be of independent intervention for the pair $(A, B)$ if the noise and the disturbance are independent of the object system; or precisely if there is observables $N$ and $D$ of probe $\mathbf{P}$ such that $N(A)=1 \otimes N$ and $D(B)=1 \otimes D$. In this case, we have $\left[N(A), B^{\text {in }}\right]=\left[A^{\text {in }}, D(B)\right]=0$. Therefore, we conclude that if the measuring interaction $U$ is of independent intervention for the pair $(A, B)$, the Heisenberg noise-disturbance uncertainty relation for the pair $(A, B)$, $E q$. (2), holds for any object state $\psi$ and any probe state $\xi$. The above conclusion was previously suggested in part by Braginsky and Khalili 16 with a limited justification and now fully justified.

The universally valid uncertainty relation shows that for measurements of dependent intervention, the lower bound of the noise-disturbance product depends on the pre-measurement uncertainties (standard deviations) of $A$ and $B$. In order to obtain the trade-off among the noise $\epsilon(A)$, the disturbance $\eta(B)$, and the pre-measurement uncertainties $\sigma(A)$ and $\sigma(B)$, we apply the Heisenberg uncertainty principle, Eq. (11), to all terms in the lefthand-side of the universally valid noise-disturbance uncertainty relation, Eq. (14). Then, we now obtain the generalized noise-disturbance uncertainty relation,

$$
\epsilon(A) \eta(B)+\epsilon(A) \sigma(B)+\sigma(A) \eta(B) \geq \frac{|\langle\psi|[A, B]| \psi\rangle|}{2} .
$$

The above relation holds for any apparatus $\mathbf{A}$ (specified by any probe state $\xi$, any unitary operator $U$, and any probe observable $M$ ), any observables $A, B$, and any input state $\psi$, and hence ultimately generalizes the Heisenberg noise-disturbance uncertainty relation, Eq. (2), to arbitrary measurements.

Under the finite energy constraint, i.e., $\sigma(Q), \sigma(P)<$ $\infty$, the above relation excludes the possibility of having both $\epsilon(Q)=0$ and $\eta(P)=0$. However, $\epsilon(Q)=0$ is possible with $\sigma(Q) \eta(P) \geq \hbar / 2$ and $\eta(P)=0$ is possible with $\epsilon(Q) \sigma(P) \geq \hbar / 2$. In particular, even the case where $\epsilon(Q)=0$ and $\eta(P)<\varepsilon$ with arbitrarily small $\varepsilon$ is possible for some input state with $\sigma(Q)>\hbar / 2 \varepsilon$, and also the case where $\eta(P)=0$ and $\epsilon(Q)<\varepsilon$ is possible for some input state with $\sigma(P)>\hbar / 2 \varepsilon$. Such extreme cases occur in compensation for large uncertainties in the input state, while in the minimum uncertainty state with $\sigma(Q)=$ $\sigma(P)=(\hbar / 2)^{1 / 2}$, we have

$$
\epsilon(Q) \eta(P)+\sqrt{\frac{\hbar}{2}}[\epsilon(Q)+\eta(P)] \geq \frac{\hbar}{2} .
$$

Even in this case, it is allowed to have $\epsilon(Q) \eta(P)=0$ with $\epsilon(Q)=0$ and $\eta(P) \geq(\hbar / 2)^{1 / 2}$ or with $\eta(P)=0$ and $\epsilon(Q) \geq(\hbar / 2)^{1 / 2}$.

\section{VIOLATION OF THE HEISENBERG INEQUALITY}

Now let us consider the problem as to whether one can implement, under the current experimental technique, a good measuring apparatus with small noisedisturbance product beyond the original Heisenberg lower bound. The controversy 117 on the sensitivity limit of gravitational wave detectors suggested that the Heisenberg noise-disturbance uncertainty relation is not universally valid. In fact, based on the Heisenberg noisedisturbance uncertainty relation, Braginsky, Caves, and others [18, 19] claimed that there is a sensitivity limit, called the standard quantum limit (SQL), for monitoring the free-mass position that leads to a quantum mechanical sensitivity limit on interferometer type gravitational wave detectors. However, Yuen 20 proposed the idea of "contractive state measurements" to break the SQL and Ozawa 21, 22 found an explicit Hamiltonian realization of a contractive state measurement that breaks the SQL. Consequently, the above measuring interaction violates the Heisenberg noise-disturbance uncertainty relation. Direct computaions on the position-measuringniose and momentum-disturbance was given in [23] showing the violation of the Heisenberg noise-disturbance uncertainty relation. 
In what follows, modifying the above interaction in the context of optical quadrature measurement, I will show that the small noise-disturbance product can be achieved beyond Heisenberg's lower bound by an apparatus carrying out a precise and nearly non-disturbing quadrature measurement with currently available linear optical devices.

\section{BACKACTION EVADING QUADRATURE AMPLIFIERS}

Consider the case where the system $\mathbf{S}$ and the probe $\mathbf{P}$ are two optical modes represented by annihilation operators $a$ and $b$, respectively. The quadrature component field operators $X_{a}, Y_{a}, X_{b}, Y_{b}$ are self-adjoint operators satisfying $a=X_{a}+i Y_{a}$ and $b=X_{b}+i Y_{b}$, for which we have $\left[X_{a}, Y_{a}\right]=i / 2$ and $\left[X_{b}, Y_{b}\right]=i / 2$.

A measuring interaction $U$ on $\mathbf{S}+\mathbf{P}$ is called a backaction evading (BAE) quadrature amplifier [24] with gain $G$ if we have

$$
\begin{aligned}
& X_{a}^{\text {out }}=X_{a}^{\text {in }}, \\
& X_{b}^{\text {out }}=X_{b}^{\text {in }}+G X_{a}^{\text {in }}, \\
& Y_{a}^{\text {out }}=Y_{a}^{\text {in }}-G Y_{b}^{\text {in }}, \\
& Y_{b}^{\text {out }}=Y_{b}^{\text {in }} .
\end{aligned}
$$

In order to measure $X_{a}$, the probe observable is chosen best to be $M=X_{b} / G$. Then we have

$$
M^{\text {out }}=X_{a}^{\mathrm{in}}+\frac{1}{G} X_{b}^{\mathrm{in}} .
$$

The $X_{a}$-noise operator, the $X_{a}$-disturbance operator, and the $Y_{a}$-disturbance operator are given by

$$
\begin{aligned}
N\left(X_{a}\right) & =\frac{1}{G} X_{b}^{\mathrm{in}}, \\
D\left(X_{a}\right) & =0, \\
D\left(Y_{a}\right) & =-G Y_{b}^{\mathrm{in}} .
\end{aligned}
$$

The condition $D\left(X_{a}\right)=0$ is characteristic of BAE amplifiers. If the probe is prepared nearly in the $X_{b}$ eigenstate $\left|X_{b}=0\right\rangle$, the measurement is a nearly noiseless $\left(\epsilon\left(X_{a}\right) \approx 0\right)$ and nondisturbing $\left(\eta\left(X_{a}\right)=0\right)$ measurement of $X_{a}$. From the above, BAE amplifiers are of independent intervention for the pair $\left(X_{a}, Y_{a}\right)$. Thus, the Heisenberg noise-disturbance uncertainty relation for the pair $\left(X_{a}, Y_{a}\right)$ holds. If the $b$-mode is prepared in the vacuum, $\xi=|0\rangle$, the noise and disturbance satisfy $\epsilon\left(X_{a}\right)=1 / 2$ and $\eta\left(Y_{a}\right)=1 / 2$, so that the minimum noise-disturbance product attains as $\epsilon\left(X_{a}\right) \eta\left(Y_{a}\right)=1 / 4$.

\section{NOISELESS QUADRATURE TRANSDUCERS}

Consider the following input-output relations

$$
\begin{aligned}
& X_{a}^{\text {out }}=X_{a}^{\text {in }}-X_{b}^{\text {in }}, \\
& X_{b}^{\text {out }}=X_{a}^{\text {in }} \\
& Y_{a}^{\text {out }}=-Y_{b}^{\text {in }} \\
& Y_{b}^{\text {out }}=Y_{b}^{\text {in }}+Y_{a}^{\text {in }} .
\end{aligned}
$$

In this case, the measuring interaction $U$ is called the noiseless quadrature transducer. In order to measure $X_{a}$, the probe observable is chosen to be $M=X_{b}$. The $X_{a^{-}}$ noise operator, the $X_{a}$-disturbance operator, and the $Y_{a^{-}}$ disturbance operator are given by

$$
\begin{aligned}
N\left(X_{a}\right) & =0 \\
D\left(X_{a}\right) & =-X_{b}^{\mathrm{in}}, \\
D\left(Y_{a}\right) & =-\left(Y_{a}^{\mathrm{in}}+Y_{b}^{\mathrm{in}}\right) .
\end{aligned}
$$

The condition $N\left(X_{a}\right)=0$ is characteristic of the noiseless transducer. Hence, the measurement is a noiseless $X_{a^{-}}$ measurement regardless of the probe preparation $\xi$. From $(21 \mathrm{~b})$, if the probe is prepared nearly in the $X_{b}$ eigenstate $\left|X_{b}=0\right\rangle$, the measurement is a noiseless $\left(\epsilon\left(X_{a}\right)=0\right)$ and nearly nondisturbing $\left(\eta\left(X_{a}\right) \approx 0\right)$ measurement of $X_{a}$. Since $\epsilon\left(X_{a}\right)=0$, we have

$$
\epsilon\left(X_{a}\right) \eta\left(Y_{a}\right)=0
$$

for any states $\psi$ and $\xi$, so that Heisenberg's lower bound for the noise-disturbance product can be overcome by a noiseless and nearly nondisturbing quadrature measurement, if one can implement a noiseless quadrature transducer. The above model also suggests that the linearity of measuring interaction does not ensure the validity of the Heisenberg noise-disturbance uncertainty relation, despite a prevailing claim that linear measurements, measurements closely connected to linear systems, obey the Heisenberg noise-disturbance uncertainty relation [16].

\section{EXPERIMENTAL REALIZATION OF THE NOISELESS QUADRATURE TRANSDUCERS}

The noiseless quadrature transducer can be implemented, in principle, as follows. Consider two degenerate modes $a$, the signal, and $b$, the probe, with frequency $\omega$ and orthogonal polarization, which undergo successive parametric interactions in the following 5 steps; see Refs. [24 28] for similar implementations of BAE amplifiers.

(i) The two polarization modes undergo a mixing interaction using a polarization rotator which rotates the angle of polarization by $\theta$. The operation of the polarization rotator is represented by the mixing operator

$$
T(\theta)=\exp \left[\theta\left(a b^{\dagger}-a^{\dagger} b\right)\right]
$$

(ii) The mixture of the signal and the probe fields will propagate along each of the ordinary and orthogonal extraordinary axis of a KTP crystal pumped by a pulsed intense classical field. This interaction is a nondegenerate 
parametric amplifier described by the two-mode squeeze operator

$$
S(r)=\exp \left[r\left(a b-a^{\dagger} b^{\dagger}\right)\right]
$$

where $r$ corresponds to the squeezing parameter.

(iii) After the amplification step the fields pass through a second polarization rotator with the mixing angle $2 \theta$ so that the operation is represented by $T(2 \theta)=T(\theta)^{2}$.

(iv) The mixture of the signal and the probe fields undergoes the second parametric amplification described by the two-mode squeeze operator $S(-r)$.

(v) After the second amplification step the field pass through a third polarization rotator with the mixing angle $\theta$ so that the operation is represented by $T(\theta)$.

Thus this process defines the measuring interaction operator

$$
U(r, \theta)=T(\theta) S(-r) T(\theta) T(\theta) S(r) T(\theta) .
$$

We shall determine the parameters $\theta$ and $r$ for $U(r, \theta)$ to realize a noiseless quadrature transducer, Eq. (20). Suppose that $\theta$ and $r$ satisfies the relation $\sin 2 \theta=\tanh r$. In this case, it is well-known 24 28] that the unitary operator $U_{-}=T(\theta) S(-r) T(\theta)$ realizes the BAE quadrature amplifier with $G=2 \sinh r$, i.e.,

$$
\begin{aligned}
& U_{-}^{\dagger} X_{a} U_{-}=X_{a}, \\
& U_{-}^{\dagger} X_{b} U_{-}=X_{b}+2(\sinh r) X_{a}, \\
& U_{-}^{\dagger} Y_{a} U_{-}=Y_{a}-2(\sinh r) Y_{b}, \\
& U_{-}^{\dagger} Y_{b} U_{-}=Y_{b} .
\end{aligned}
$$

Similarly, the unitary operator $U_{+}=T(\theta) S(r) T(\theta)$ realizes the conjugate BAE quadrature amplifier, i.e.,

$$
\begin{aligned}
U_{+}^{\dagger} X_{a} U_{+} & =X_{a}-2(\sinh r) X_{b}, \\
U_{+}^{\dagger} X_{b} U_{+} & =X_{b}, \\
U_{+}^{\dagger} Y_{a} U_{+} & =Y_{a}, \\
U_{+}^{\dagger} Y_{b} U_{+} & =Y_{b}+2(\sinh r) Y_{a} .
\end{aligned}
$$

Combining the above equations, we have the inputoutput relations for the unitary operator $U(r, \theta)=$ $U_{-} U_{+}$,

$$
\begin{aligned}
U(r, \theta)^{\dagger} X_{a} U(r, \theta) & =X_{a}-2(\sinh r) X_{b}, \\
U(r, \theta)^{\dagger} X_{b} U(r, \theta) & =\left(1-4 \sinh ^{2} r\right) X_{b}+2(\sinh r) X_{a}, \\
U(r, \theta)^{\dagger} Y_{a} U(r, \theta) & =\left(1-4 \sinh ^{2} r\right) Y_{a}-2(\sinh r) Y_{b}, \\
U(r, \theta)^{\dagger} Y_{b} U(r, \theta) & =Y_{b}+2(\sinh r) Y_{a} .
\end{aligned}
$$

Thus, if $\sinh r=1 / 2$, i.e.,

$$
\begin{aligned}
& r=\log \frac{1+\sqrt{5}}{2} \\
& \theta=\frac{1}{2} \sin ^{-1} \frac{1}{\sqrt{5}},
\end{aligned}
$$

the resulting unitary operator $U=U(r, \theta)$ realizes the noiseless quadrature transducer, Eq. (20).

Thus, if $r$ and $\theta$ are chosen as Eq. (29), the parametric process (i)-(v) realizes the noiseless quadrature transducer. Therefore, this process followed immediately by the homodyne detection of the $X_{b}$ component implements a nearly nondisturbing and noiseless measurement of the $X_{a}$ quadrature component that disturbs the conjugate observable $Y_{a}$ much less than the quantum limit set by Heisenberg's lower bound for the noise-disturbance product, $\epsilon\left(X_{a}\right) \eta\left(Y_{a}\right) \geq 1 / 4$.

\section{CONCLUSION}

In this paper, I have proposed new relations, (14) and (15), universally valid for the trade-off between the measurement noise and disturbance. These relations demonstrates that the prevailing Heisenberg's lower bound for the noise-disturbance product is valid for measurements with independent intervention, but can be circumvented by a measurement with dependent intervention. An experimental confirmation of the violation of Heisenberg's lower bound is proposed for a measurement of optical quadrature with currently available techniques in quantum optics. The new relation will not only bring a new insight on fundamental limitations on measurements set by quantum mechanics but also advance a frontier of precision measurement technology such as gravitational wave detection and quantum information processing.

\section{ACKNOWLEDGMENTS}

Acknowledgments. The author thanks F. De Martini, S. Takeuchi, and I. Ojima for useful discussions. This work was supported by the programme "R\&D on Quantum Communication Technology" of the MPHPT of Japan, by the CREST project of the JST, and by the Grant-in-Aid for Scientific Research of the JSPS.

[1] Heisenberg, W. Über den anschaulichen Inhalt der quantentheoretischen Kinematik und Mechanik. Z. Phys. 43, 172-198 (1927); The physical content of quantum kinematics and mechanics (Engl. Trans.), in Quantum Theory and Measurement (eds Wheeler, J. A. \& Zurek, W. H.) (Princeton Univ. Press, Princeton, 1983).

[2] Heisenberg, W. The Physical Principles of the Quantum Theory, (Univ. of Chicago Press, Chicago, 1930; Dover, New York, 1949, 1967).

[3] Kennard, E. H. Zur quantenmechanik einfacher bewegungstypen. Z. Phys. 44, 326-352 (1927). 
[4] Robertson, H. P. The uncertainty principle. Phys. Rev. 34, 163-164 (1929).

[5] Ballentine, L. E. The statistical interpretation of quantum mechanics. Rev. Mod. Phys. 42, 358-381 (1970).

[6] Arthurs, E. \& Kelly, Jr., J. L. On the simultaneous measurement of a pair of conjugate observables. Bell. Syst. Tech. J. 44, 725-729 (1965).

[7] Arthurs, E. \& Goodman, M. S. Quantum correlations: A generalized Heisenberg uncertainty relation. Phys. Rev. Lett. 60, 2447-2449 (1988).

[8] Ozawa, M. Quantum limits of measurements and uncertainty principle, in Quantum Aspects of Optical Communications (eds Bendjaballah, C., Hirota, O. and Reynaud, S.) 3-17 (Springer, Berlin, 1991).

[9] Ishikawa, S. Uncertainty relations in simultaneous measurements for arbitrary observables. Rep. Math. Phys. 29, 257-273 (1991).

[10] She, C. Y. \& Heffner, H. Simultaneous measurement of noncommuting observables. Phys. Rev. 152, 1103-1110 (1966).

[11] Yuen, H. P. Generalized quantum measurements and approximate simultaneous measurements of noncommuting observables. Phys. Lett. A 91, 101-104 (1982).

[12] Yamamoto, Y. \& Haus, H. A. Preparation, measurement and information capacity of optical quantum states. Rev. Mod. Phys. 58, 1001-1020 (1986).

[13] Ozawa, M. Measurements of nondegenerate discrete observables. Phys. Rev. A 62, 062101(1-13) (2000).

[14] Ozawa, M. Operations, disturbance, and simultaneous measurability. Phys. Rev. A 63, 032109(1-15) (2001).

[15] Ozawa, M. Conservation laws, uncertainty relations, and quantum limits of measurements. Phys. Rev. Lett. 88, 050402(1-4) (2002).

[16] Braginsky, V. B. \& Khalili, F. Y. Quantum Measurement (Cambridge Univ. Press, Cambridge, 1992): Chapter V.

[17] Maddox, J. Beating the quantum limits. Nature (London) 331, 559-559 (1988).

[18] Braginsky, V. B. \& Vorontsov, Y. I. Quantum-mechanical limitations in macroscopic experiments and modern experimental technique. Usp. Fiz. Nauk 114, 41-53 (1974) [Sov. Phys. Usp. 17, 644-650 (1975)].

[19] Caves, C. M., Thorne, K. S., Drever, R. W. P., Sandberg, V. D. \& Zimmermann., M. On the measurement of a weak classical force coupled with a quantum mechanical oscillator, I, Issues of principle. Rev. Mod. Phys. 52, 341392 (1980).

[20] Yuen, H. P. Contractive states and the standard quantum limit for monitoring free-mass positions. Phys. Rev. Lett. 51, 719-722 (1983).

[21] Ozawa, M. Measurement breaking the standard quantum limit for free-mass position. Phys. Rev. Lett. 60, 385-388 (1988).

[22] Ozawa, M. Realization of measurement and the standard quantum limit, in Squeezed and Nonclassical Light (eds P. Tombesi and E. R. Pike) 263-286 (Plenum, New York, 1989).

[23] Ozawa. M. Position measuring interactions and the Heisenberg uncertainty principle. Phys. Lett. A 299, 1-7 (2002).

[24] Yurke, B. Optical back-action-evading amplifiers. J. Opt.
Soc. Am. B 2, 732-738 (1985).

[25] La Porta, A., Slusher, R. E. \& Yurke, B. Back-action evading measurements of an optical field using parametric down conversion. Phys. Rev. Lett. 62, 28-31 (1989).

[26] Song, S., Caves, C. M., \& Yurke, B. Generation of superposition of classically distinguishable quantum states from optical back-action evasion. Phys. Rev. A 41, 52615264 (1990).

[27] Pereira, S. F., Ou Z. Y., \& Kimble, H. J. Backaction evading measurements for quantum nondemolition detection and quantum optical tapping. Phys. Rev. Lett. 72, 214217 (1994).

[28] Grangier, P., Levenson, J. A. \& Poiszat, J-P. Quantum non-demolition measurements in optics. Nature (London) 396, 537-542 (1998). 